\title{
Stability Analysis of Roundness Profiles of Drawn Tubes
}

Augustín Görög, Ingrid Görögová

Slovak University of Technology in Bratislava, Faculty of Material Science and Technology in Trnava, Institute of Production Technologies, J. Bottu 25, 91724 Trnava. Slovak Republic. E-mail: augustin.gorog@stuba.sk, ingrid.gorogova@stuba.sk

The geometric accuracy is significant property of the product. For semi-finished products, this accuracy may also affect the accuracy of the final product (the resulting mechanical component). Geometric accuracy (inaccuracy) can be transmitted from one operation to the next in production - technological heredity arises. It is, therefore, essential to analyze the deviations on the produced areas and take measures to ensure that the negative effect of one operation was not transferred to the next operation. It is insufficient to analyze only the numerical values of the measured deviations. It is necessary to directly analyze the measured profiles in the field of macro geometry. The paper presents a stability analysis of roundness profiles measured on the drawn tube. The analysis uses knowledge from harmonic analysis and from the Fourier series. The measured roundness profiles are divided into individual harmonic components. Arithmetic means of amplitudes of individual harmonic components are monitored. There were analyzed parameters determining the stability of the profile - standard deviations.

Keywords: drawn tubes, technological heredity, roundness profiles, harmonic analysis, Fourier series

\section{Introduction}

Tube drawing is a well-known technological process. Is used for the reduction of diameter and wall thickness to obtain specific dimensions. The primary tube is drawn into a die with a smaller hole and its thickness achieved by use of a mandrel. [1] Due to versatility and good mechanical properties of the arts, drawing process is one of the most used metal-forming processes in the industrial field. The materials traditionally used in manufacturing processes are steels, aluminum and copper alloys. [2,3] The profiles of drawing die orifice and the mandrel are key factors to achieve the requirements of tube geometric precision and the surface roughness. [4] The circular and cylindrical features are fundamental geometric features in machines. The fitting conditions of cylindrical components have a significant effect on the performance of precision products, and they are determined by size tolerances and geometrical errors such as cylindricity error of the mating parts. Currently, the fitting conditions are mostly estimated by the size tolerances and the geometrical errors are normally ignored. [5]

\section{Harmonic analysis and importance of fil- tration}

Nowadays a geometrical surface structure is usually evaluated with the use of Fourier transform. This type of transform allows for accurate analysis of harmonic components of surface profiles. Due to its fundamentals, Fourier transform is particularly efficient when evaluating periodic signals. [6] Fourier function is used to identify the harmonics of the components in the measured data. The harmonic analysis is one of the most frequently applied methods for mathematical representation of form profiles of cylindrical surfaces. It is enabling, for any profile, the determination of a particular number of harmonics (amplitudes and phase shifts). Autor Nodrzykowski [7] presented measuring procedures and principles of determining deviations of shape and location of the crankshaft main necks assembly axis. He also described mathematical methods of roundness shape description based on the harmonic analysis theory for the cases when the shaft is set with the external journals in prisms and for the case when the shaft is set on the external front faces in the claws. Authors Sogalad and Udupa [8] in their article made a detailed study of the influence of harmonics and the average undulation number of roundness profiles on the load bearing ability of interference-fitted assemblies. For this purpose, a plan of experiments was prepared by taking 32 factorial design with interference and the average undulation number as parameters. The analysis revealed that both the parameters influence the load bearing ability of interference-fitted assemblies. Further, it is found that harmonics influence the load bearing ability of interference-fitted assemblies. Denis and Samuel [9] suggested a harmonic analysis method to identify and separate the dominant harmonic components in the form profile of the spindle. A mathematical description of the proposed method was described and experimental results were presented. The application of the proposed method to the evaluation of the synchronous radial error of a highspindle was provided for measured data. The proposed method analyzes the data measured in the time do- 
main and is suitable for the identification of the spindle errors at high-speed conditions. The couples comparing method [10] is a new concept that permits comparison of roundness profiles. This idea, owing to its methodology, allows comparison of roundness profiles obtained with the use of the V-block method with the ones obtained by the radius change method. It is also possible to adjust the couples comparison method to determine optimum parameters of measuring instruments as well as to comparing measuring instruments. Then, it can compare values of coefficients of expansion of profiles into trigonometric Fourier series, amplitudes of analyzed harmonic components as well their phase shifts. The roundness measuring instrument measures the deviations of points on the surface from the ideal circle on the perimeter of the component. The number of measuring points on the perimeter of the component may vary and depends on the capabilities of the roundness measuring instrument. It is usually measured e.g. 1800 or 3600 points. These points will show the measured profile in a roundness graph. This profile represents both macrogeometric and microgeometric deviations. In order to separate the macrogeometry from the microgeometry, it is necessary to use filtration. Filtration divides the profile
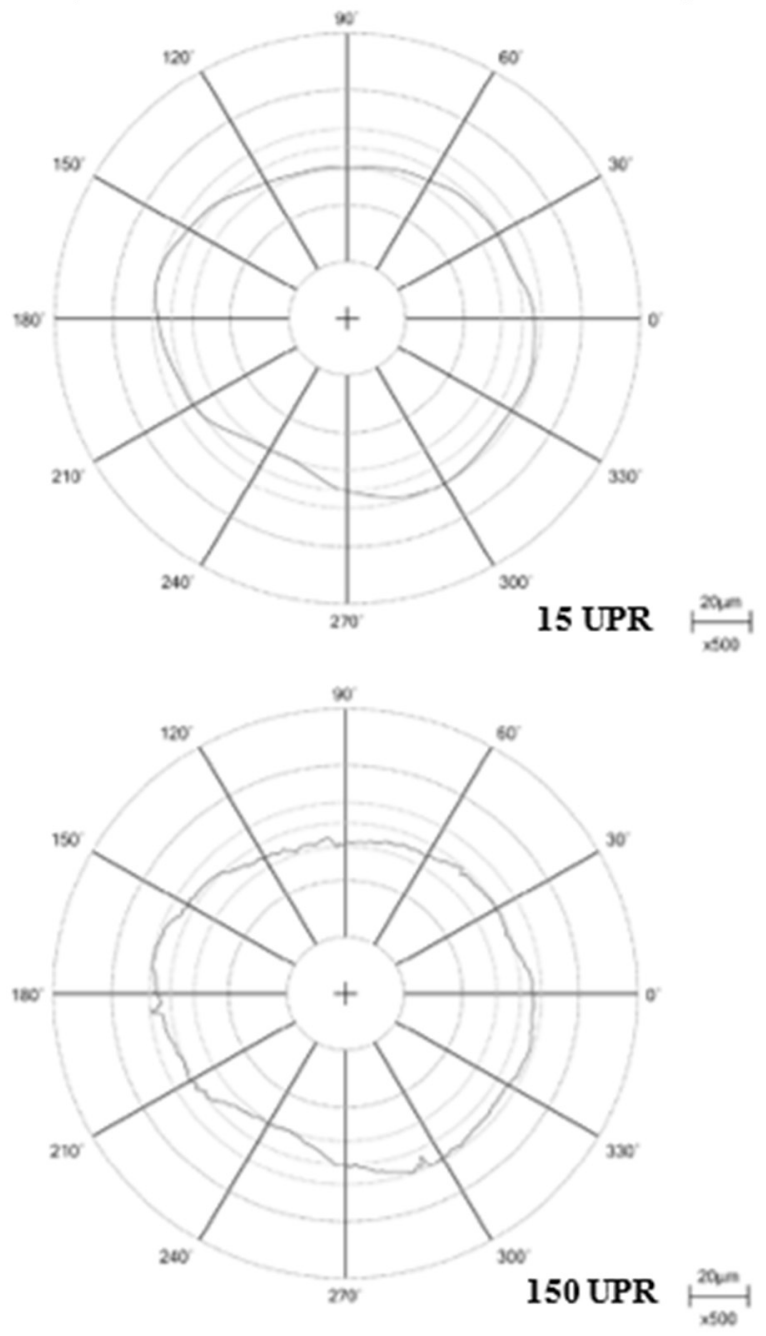

into individual harmonic components and suppresses some (decreases, eliminates) as needed. The use of filtering is referred e.g.: Gauss low 50 UPR. Gaus refers to the mathematical procedure (mathematical formulas) by which the filtration was calculated. Low means that low wavelength waves have been suppressed. UPR value indicates the number of waves on the perimeter. It is the boundary that determines which waves are considered in the profile. This value is most often set to 15 UPR, 50 UPR, 150 UPR or 500 UPR. It depends on the diameter of the area on which roundness is measured. A smaller UPR is used on a smaller diameter and a larger UPR is used on a larger diameter. Fig. 1 presents the measured roundness profile. A Gauss low filter was used. The UPR value has been changed - 15, 50, 150 and 500 UPR. Using a different UPR value changes the profile and the roundness, too $(\mathrm{RON})$ :

15 UPR: $\mathrm{RON}=13.641 \mu \mathrm{m}$

50 UPR: $\mathrm{RON}=14.661 \mu \mathrm{m}$

150 UPR: $\mathrm{RON}=15.396 \mu \mathrm{m}$

500 UPR: $\mathrm{RON}=17.254 \mu \mathrm{m}$

Since the diameter of the measured area was 25 $\mathrm{mm}$, it is advisable to use a 50 UPR filter (wavelength $1.57 \mathrm{~mm})$.
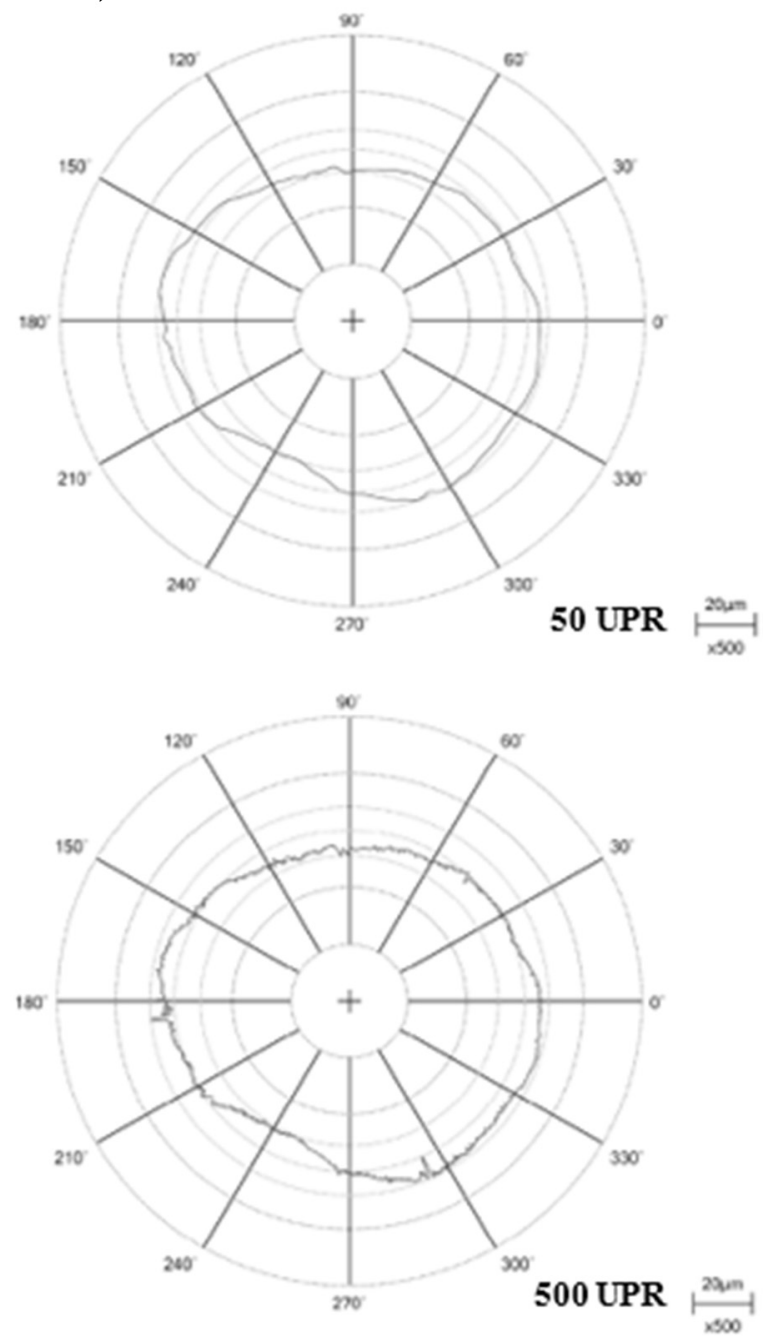

Fig. 1 Using a Gauss low filter with different UPRs 


\section{Methodology of experiment}

The aim of the experimental work was to determine the stability of roundness profiles of drawn tubes. That is, how the roundness and the roundness profile itself change on the drawn tube in different cross-sections. If the tube will have an elliptical roundness profile in one measured section, it is necessary to determine whether it will have a roundness profile of the same shape in another section (eg. in cross-section, which is $50 \mathrm{~mm}$ below the first). The aim is to compare the roundness (measured on one tube) from the measured roundness values, but also the point of view of the shape of the measured roundness profiles. Drawn tubes are produced by drawing through a die, the quality of which greatly affects the macrogeometric shape of the tube. Of course, several technological factors - conditions under which drawing takes place (lubrication, drawing speed, tube wall thickness...) enter the production. The experimental work consisted of measuring circular profiles on four drawn tubes, which are from two manufacturers. The tube A was made of steel STN 41 1353. Its diameter was $\phi 28 \mathrm{~mm}$ and the wall thickness was 4 $\mathrm{mm}$. Tubes B, C and D were made of E235 and were from a different manufacturer (i.e. the drawing was performed under conditions other than drawing of tube A). The last operation in their production was drawing through a die from the original size (diameter $33.8 \mathrm{~mm}$, wall thickness $3 \mathrm{~mm}$ ) to the new size (diameter $24 \mathrm{~mm}$, wall thickness $2.5 \mathrm{~mm}$ ). All tubes had the same dimensions. There are various devices for measuring of roundness. [11] Roundness was measured on a RONDCOM 60A instrument (Accretech, Japan). The parameters for roundness measurement (on the outer surfaces) and evaluation were as follows:

- Number of measured points by roundness measurement: 3600

- Method of roundness evaluation: MZC (The Minimum Zone Circle) [12, 13]

- The filter used to evaluate the roundness: Gauss low 50 UPR

- Number of measured cross-sections on the tube: 20

- Distance between cross-sections: $5 \mathrm{~mm}$

- Measured length: $95 \mathrm{~mm}$

Tab. 1 presents the measured values of roundness in individual cross-sections on tubes $\mathrm{A}, \mathrm{B}, \mathrm{C}$ and $\mathrm{D}$. At the bottom of the table there are quantified arithmetic values and basic indicators of the variance of values. It can be stated the standard deviations are small compared to the average values and the roundness value is relatively stable.
Tab. 1 Measured values of roundness [um]

\begin{tabular}{|c|c|c|c|c|}
\hline $\begin{array}{l}\text { Cross- } \\
\text { section }\end{array}$ & Tube A & Tube B & Tube C & Tube D \\
\hline 1 & 44.099 & 35.639 & 22.008 & 12.969 \\
\hline 2 & 42.579 & 35.551 & 28.286 & 13.419 \\
\hline 3 & 44.024 & 35.445 & 31.575 & 13.584 \\
\hline 4 & 47.535 & 35.090 & 32.827 & 14.661 \\
\hline 5 & 44.076 & 35.716 & 34.187 & 15.576 \\
\hline 6 & 43.773 & 34.820 & 34.303 & 14.369 \\
\hline 7 & 44.057 & 36.256 & 35.648 & 13.124 \\
\hline 8 & 44.128 & 33.269 & 37.253 & 12.742 \\
\hline 9 & 43.133 & 26.843 & 37.909 & 13.207 \\
\hline 10 & 42.263 & 32.391 & 38.437 & 13.878 \\
\hline 11 & 43.002 & 37.020 & 38.014 & 18.669 \\
\hline 12 & 43.881 & 32.392 & 36.385 & 15.698 \\
\hline 13 & 42.643 & 38.141 & 36.481 & 15.936 \\
\hline 14 & 42.455 & 31.471 & 36.115 & 18.982 \\
\hline 15 & 43.383 & 33.205 & 36.393 & 20.407 \\
\hline 16 & 49.570 & 30.296 & 35.358 & 22.629 \\
\hline 17 & 42.662 & 23.942 & 34.612 & 17.756 \\
\hline 18 & 41.701 & 30.681 & 34.332 & 16.302 \\
\hline 19 & 42.953 & 34.441 & 34.619 & 15.571 \\
\hline 20 & 42.121 & 31.075 & 35.011 & 16.375 \\
\hline Average & 43.702 & 33.585 & 34.488 & 15.793 \\
\hline Max & 49.570 & 38.141 & 38.437 & 22.629 \\
\hline Min & 41.701 & 23.942 & 22.008 & 12.742 \\
\hline $\begin{array}{c}\text { Variation } \\
\text { range }\end{array}$ & 7.869 & 14.199 & 16.429 & 9.887 \\
\hline $\begin{array}{l}\text { Standard } \\
\text { deviation }\end{array}$ & 1.849 & 3.188 & 3.764 & 2.711 \\
\hline
\end{tabular}

\section{Analysis of roundness profiles}

The roundness profiles were also monitored. Fig. 2 shows the roundness profiles of the individual tubes (the figures have the same scale). On each image are collectively displays all profiles measured on individual tubes. All profiles measured on one pipe are very similar.

Harmonic analysis was performed on the measured roundness profiles. Harmonic analysis was performed on the measured roundness profiles. The aim was to determine the amplitudes of the individual harmonic components and to determine whether the values of these amplitudes on the tubes are stable. FIG. 3 graphically presents the values of the amplitudes of the individual tubes. Average values are given here, which were calculated as the arithmetic mean of all measured profiles (tubes $\mathrm{A}, \mathrm{B}, \mathrm{D}=20$ profiles, tube $\mathrm{C}$ $=15$ profiles). At the same time, the standard deviation is displayed graphically at each amplitude, indicating the variance of the individual amplitudes. There are shown only components from 2 UPR to 15 UPR. Amplitudes with greater UPR have already been low. 

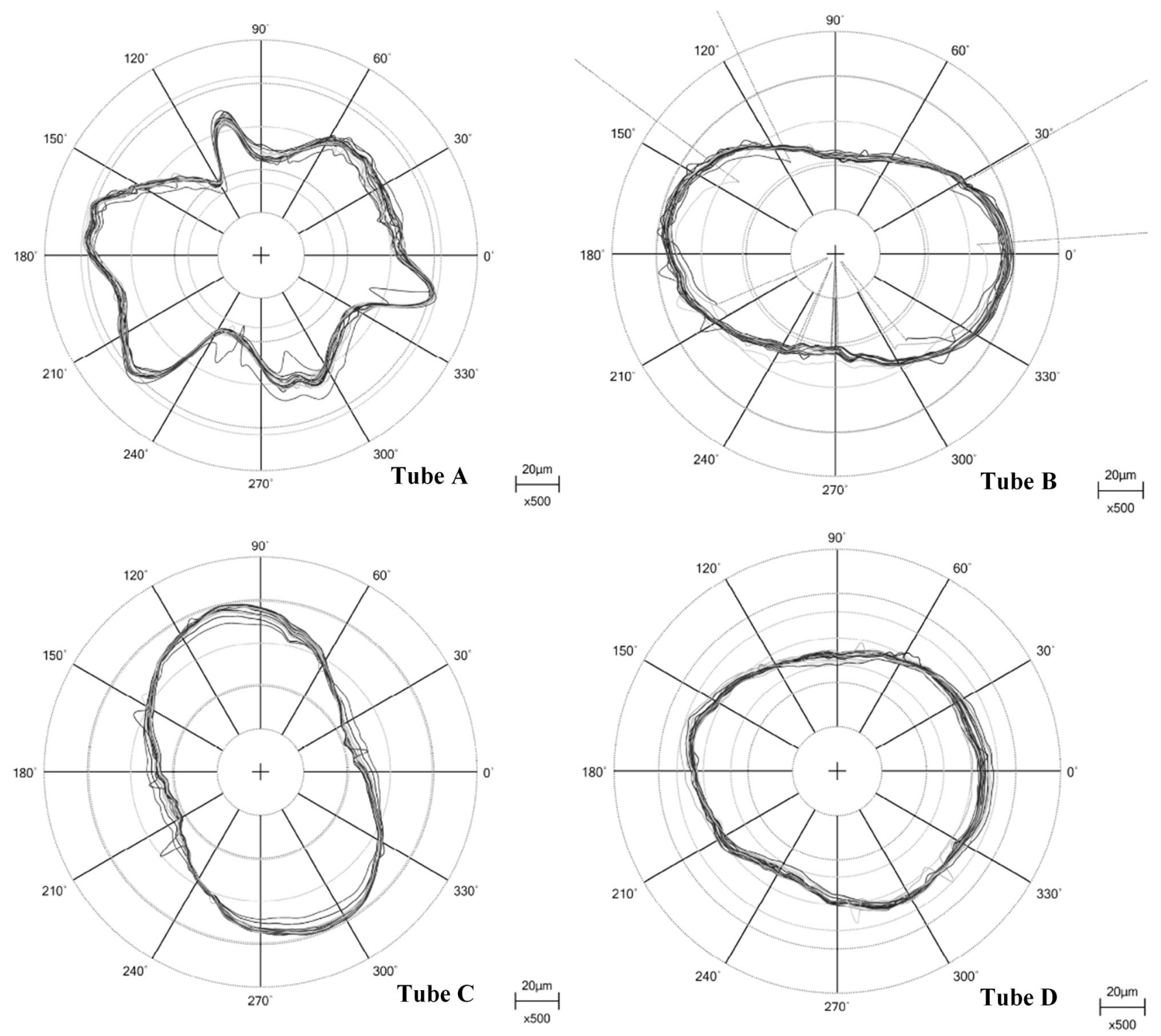

Fig. 2 Measured roundness profiles on individual tubes

The amplitudes of the second harmonic component are dominant on all measured tubes (A, B, C, D). Other harmonic components on tubes $\mathrm{B}$ and $\mathrm{C}$ are already small compared to the second harmonic component. Slightly pronounced is the third harmonic component on tube D. It is evident from the graphical representation of the roundness profile. There is dominated not only by the elliptical shape but also by the triangle shape. On tube $\mathrm{A}$, in addition to the second harmonic component the 3rd, 6th, 9th, 12th and 15th harmonic components are also dominant. All these components are a multiple of 3 , the largest of which is the 6th harmonic component. The elliptical and hexagonal shape is dominant in the profile of the tube A, which also affects other harmonic components with a multiple of 3 . The standard deviations indicate that the amplitudes of the individual harmonic components are predominantly stable. The individual measured profiles have similar amplitude results determined by harmonic analysis. It can be stated the roundness profile is stable on individual tubes - a very similar profile was measured in individual cross-sections on the tube. There was various roundness measured on tubes A, B, $\mathrm{C}$ and $\mathrm{D}$. The smaller roundness, the amplitude values of the harmonic components will be smaller and conversely - the larger roundness, the amplitudes will be larger. The individual amplitudes of the harmonic components were given to ratio with the measured roundness. The percentage is shown graphically in fig. 4. The figure confirms the previous claim that the 2nd harmonic component is dominant - the ratio of the amplitude 2nd harmonic component to the roundness is 25 to 45 percent. Other harmonic components have a significantly lesser effect, except for 3rd harmonic component on tube $\mathrm{D}$ and 6th harmonic component on tube $\mathrm{A}$. 


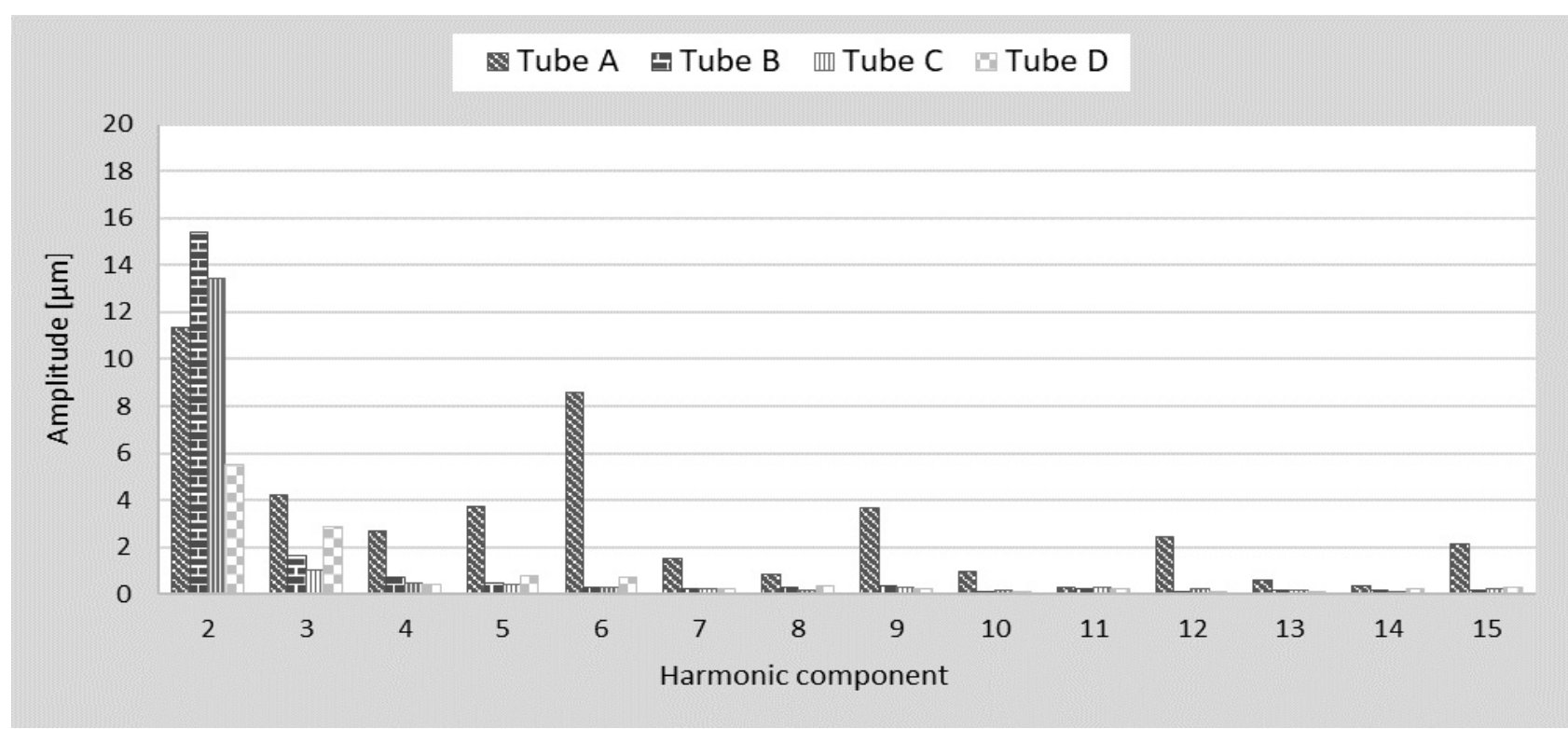

Fig. 3 Amplitude of roundness profiles on tubes

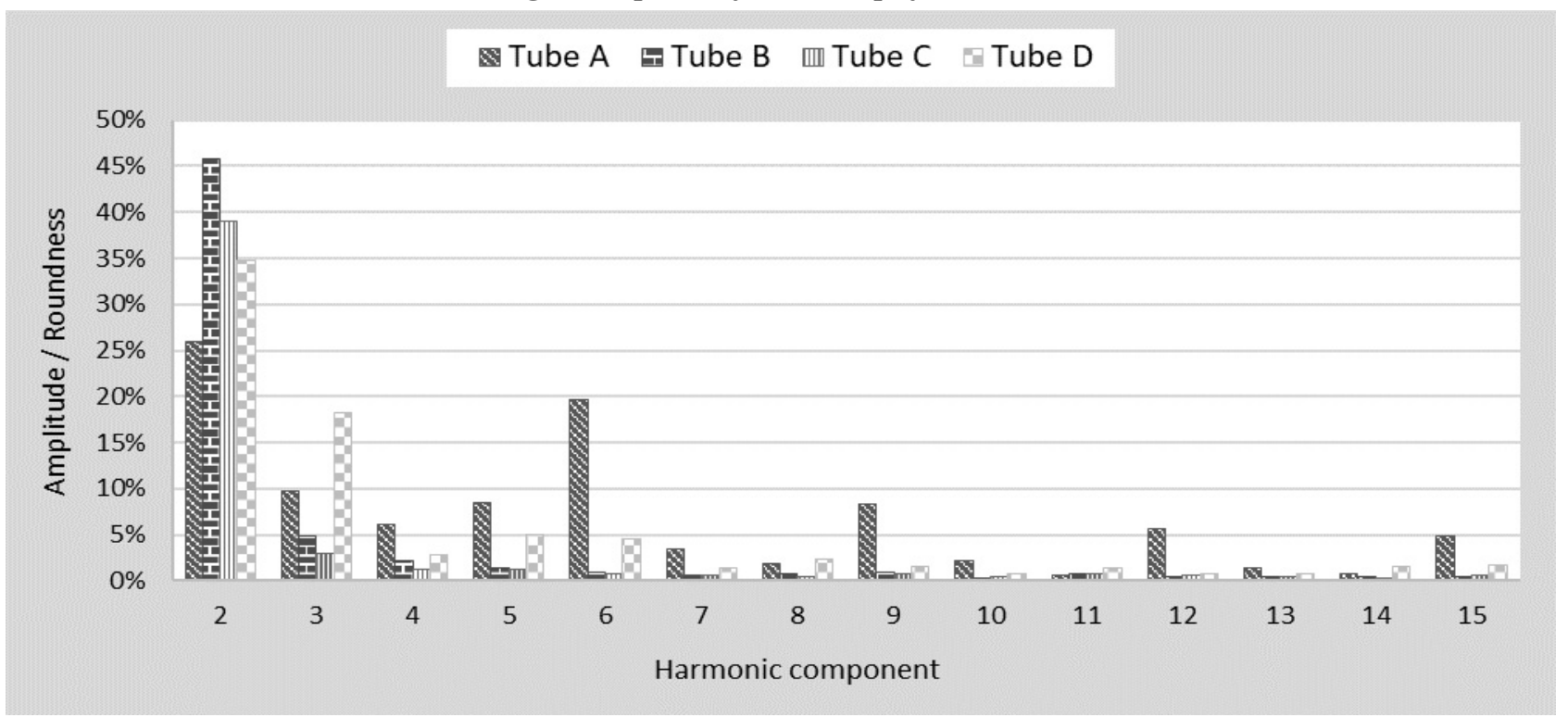

Fig. 4 Percentage ratio of amplitudes and roundness

Phase shifts cannot be compared to each other between tubes because it is the displacement of the individual harmonic components relative to the tube rotation during measurement. There were to each other compared only phase shiftss measured on one tube - on each tube were compared the phase shiftss measured in individual cross-sections (pipe A, B, D = 20 profiles, pipe $C=15$ profiles). However, the dominant second harmonic component had a small standard deviation of phase shifts from $1.7^{\circ}$ to $2.3^{\circ}$ on all tubes. For other harmonic components, the standard deviations of the phase shifts were usually larger, even though they had a significantly lower arithmetic mean. It can be stated that the standard deviations of the phase shiftss were more marked than the standard deviations of the amplitudes. Phase shiftss are therefore less stable than amplitudes.

\section{Summary}

Based on conducted experiments and the achieved results, it is possible to acknowledge these arguments:

\section{Roundness}

Roundness vary from tube to tube. On tube D was measured significantly lower roundness. The roundness on the tube is stable - in different crosssections, the value does not fluctuate dramatically. The standard deviations of the measured values were in the range 4 to $17 \%$ of the average. The lowest standard deviation was found in the tube with the highest roundness values (tube $A$ ).

\section{Roundness profile - visual evaluation}

All round profiles measured on one tube had a very similar shape - round profiles are visually stable. - Different roundness profiles were measured on different tubes. The profiles on tubes $\mathrm{B}$ and $\mathrm{C}$ were similar - the 
elliptical shape dominated there.

Roundness profile - harmonic analysis

For all profiles dominated thesecond harmonic component, which was always the greatest amplitude value. Harmonic components 3, 6, 9, 12 and 15 also dominated on the tube $\mathrm{A}$ (they are multiples of 3 ). The amplitudes on tubes $B$ and $C$ were very similar. However, the dominant 2 harmonic component had an amplitude less by $1.9 \mu \mathrm{m}$ on the tube $\mathrm{C}$, but a higher standard deviation by $3.2 \mu \mathrm{m}$ (the amplitude value fluctuated more). The amplitudes of the tubes B, C and $\mathrm{D}$ of the individual harmonic components gradually decreased. The percentage ratio of amplitudes to roundness confirmed the greatest effect of the 2 nd harmonic component $(25.9 \%$ to $45.8 \%)$. The dominant second harmonic component had a small standard deviation of the phase shifts $\left(1.7^{\circ}\right.$ to $\left.2.3^{\circ}\right)$ on all tubes. Other harmonic components had standard deviations of phase shifts usually higher despite having a significantly lower arithmetic mean value. Phase shiftss are therefore less stable than amplitudes.

\section{Acknowledgement}

The Slovak Research and Development Agency supported this work under contracts APVV-180418 Research on causes of geometrical deviations in the production of seamless tubes and their technological inheritance with emphasis on the shape stability of precision cold drawn tubes using metrological systems.

\section{References}

[1] Girard, S., D'AMOURS, G., GUILLOT, M., RAHEM, A., FAFARD, M. (2010). Experimental Exploration of the Aluminum Tube Drawing Process for Producing Variable Wall Thickness Components used in Light Structural Applications. In: SP-2294: Advances Light Weight Materials - Aluminum, Casting Materials, and Magnesium Technology, pp. 1-9.

[2] TANG, Y., OU, D., WAN, Z., LU, L., LIAN, B. (2011). Influence of drawing process parameters on forming of micro copper tube with straight grooves. In: Transactions of Nonferrous Metals Society of China, Vol. 21, No. 10, pp. 22642269

[3] SOBOTKA, J., SOLFRONK, P., KOLNEROVA, M., KORECEK, D. (2019). Input Data Acquisition Possibilities for Numerical Simulation of Drawing Process by means of the Contact-less Optical System and Thermo-camera. In: Manufacturing Technology. 2019;19(1):144-149. doi: 10.21062/ujep/259.2019/a/12132489/MT/19/1/144.
[4] GÖRÖG, A., GÖRÖGOVÁ, I., MARTINKOVIČ, M. (2020). Analysis of the surface roughness of a tube drawn by a fixed mandrel. In: Novel Trends in Production Devices and Systems VI (NTPDS VI). TTP. Zurich.

[5] WEIHUA, N., ZHENQIANG, Y. (2013). Cylindricity modeling and tolerance analysis for cylindrical components. In: Int J Adv Manuf Technol, Vol. 64, pp. 867-874.

[6] ADAMCZAK, S., MAKIEŁA, W., STEPIEN, K. (2010). Investigating Advantages and Disadvantages of the Analysis of a Geometrical Surface Structure with the Use of Fourier and Wavelet Transform. In: Metrology and Measurement System,. Vol. 17, No. 2, pp. 233-244.

[7] NOZDRZYKOWSKI, K. (2018). Applying Harmonic Analysis in the Measurements of Geometrical Deviations of the Crankshafts - Selecting Support Conditions. In: Multidisciplinary Aspects of Production Engineering, Vol. 1, No. 1, pp. 191-195.

[8] SOGALAD, I., UDUPA, N. (2009). Load bearing ability of interference-fitted assemblies: A roundness profile analysis. In: Proceedings of The Institution of Mechanical Engineers Part C-journal of Mechanical Engineering Science, Vol. 223, No. 7, pp. 1623-1632.

[9] DENIS, S., SAMUEL, G.L. (2012). Harmonicanalysis-based method for separation of form error during evaluation of high-speed spindle radial errors. In: Proceedings of the Institution of $\mathrm{Me}$ chanical Engineers, Part B: Journal of Engineering Manufacture, Vol. 226, No. 5, pp. 837-852.

[10] ADAMCZAK, S., STEPIEŃ, S., KMIECIKSOLTYSIAK, U. (2017). A Concept of an Application of Couples Comparing Method to the Comparison of Roundness Profiles. In: Procedia Engineering, Vol. 192, pp. 4-9.

[11] VIT, J., NOVAK, M. (2018) A Roundness Machine Measuring Probe Calibration. In: Manufacturing Technology. Vol. 18(6):1053-1059. doi: 10.21062/ujep/223.2018/a/12132489/MT/18/6/1053.

[12] GÖRÖG, A., GÖRÖGOVÁ. I. (2018). Research of the influence of clamping forces on the roundness deviations of the pipes turned surface. In: Research papers Faculty of Materials Science and Technology Slovak University of Technology in Trnava, Vol. 26, No. 42, pp. 47-54.

[13] SUI, W., ZHANG, D. (2012). Four Methods for Roundness Evaluation. In: Physics Procedia, Vol. 24, pp. 2159 - 2164. 